\section{The National Diabetic Retinopathy Laser Treatment Audit: implications for clinical practice in 1998}

Of all the complications of diabetes mellitus, the most feared by patients is severe visual loss due to diabetic eye disease. Despite the introduction of many effective and audited community diabetic retinopathy screening programmes, blindness due to diabetes-related eye disease is still all too common. The important results of the National Diabetic Retinopathy Laser Treatment Audit (maculopathy and proliferative disease) undertaken by the Royal College of Ophthalmologists and published in this issue, have highlighted some key findings with implications for diabetic eye care in the United Kingdom. The audits were designed to examine the process of delivery of photocoagulation treatment for diabetic retinopathy in the UK, and included a $10 \%$ validation sample of participating units that were randomly chosen. These audits are sufficiently large, with 830 patients recruited and studied, to allow major conclusions and interpretation.

The main sources of referral to diabetic eye clinics were from the hospital physician and general practitioner (maculopathy $75.2 \%$ and proliferative $69.4 \%$ ). Although up to two-thirds of patients were referred with sight-threatening retinopathy by systematic screening, it is of concern that $12 \%$ of patients with maculopathy and $28 \%$ of patients with proliferative diabetic retinopathy are not presenting until visual symptoms are present. The audit shows that there is a clear need to implement and organise screening programmes. Overall waiting times for referral to application of laser treatment showed that $14.8 \%$ of patients with proliferative retinopathy and $17.6 \%$ with maculopathy were having to wait more than 3 months. The personnel involved in giving laser treatment for maculopathy was mainly permanent staff, with only one-fifth by doctors in training, and $95 \%$ of patients had standard focal laser treatment.
In contrast in patients with proliferative diabetic retinopathy, between $32 \%$ and $40 \%$ of patients may have less laser treatment (smaller spot size and fewer laser applications) than is recommended by the DRS and ETDRS guidelines. Why this should be so is not clear, but part of the explanation may be that laser treatment for proliferative retinopathy was performed in $60 \%$ by experienced senior ophthalmological staff and a third of patients were treated by a junior doctor in training.

Despite the large current expenditure of $8 \%$ of the total National Health Service budget on diabetic care, these important audits have stressed the need for more effective diabetic retinopathy screening, with particular emphasis on proliferative diabetic retinopathy changes, which may be more difficult to detect. Extrapolating from these audit figures, it is clear there is a significant amount of sightthreatening retinopathy (both maculopathy and also proliferative diabetic retinopathy) not being detected or treated in the UK. Although the majority of laser treatments provided for diabetic patients across the country are appropriately applied by highly trained senior staff, there are still shortfalls in the provision of service.

These data surely make the case for increased provision of effective screening for diabetic retinopathy, and of highly trained permanent staff in diabetic centres to deliver effective and prompt laser treatment. Provision of the latter should help produce more standardisation in the application of laser for proliferative diabetic retinopathy.

Overall, ophthalmologists and diabetic physicians should be encouraged by these audits to demand greater allocation of resources for the early identification and treatment of diabetic eye disease, to the proven benefit of our mutual patients.
P.M. Dodson J.M. Gibson Medical Ophthalmology Clinic Birmingham Heartlands Hospital NHS Trust Bordesley Green East Birmingham B9 5PU, UK 